\title{
The far-infrared emission of the radio-loud quasar 3C 318 (Research Note)
}

\author{
P. Podigachoski, P. D. Barthel, R. F. Peletier, and S. Steendam
}

\begin{abstract}
Kapteyn Astronomical Institute, University of Groningen, 9747 AD Groningen, The Netherlands
e-mail: podigachoski@astro.rug.nl
\end{abstract}

Received 18 September 2015 / Accepted 12 November 2015

\section{ABSTRACT}

\begin{abstract}
3C 318 , a radio-loud quasar at $z=1.574$, is a subgalactic-sized radio source, and a good test-bed for the interplay between black hole and galaxy growth in the high- $z$ Universe. Based on its IRAS, ISO, and SCUBA detections, it has long been considered as one of the most intrinsically luminous $\left(L_{\mathrm{IR}}>10^{13} L_{\odot}\right)$ infrared sources in the Universe. Recent far-infrared data from the Herschel Space Observatory reveal that most of the flux associated with $3 \mathrm{C} 318$, measured with earlier instruments, in fact comes from a bright nearby source. Optical imaging and spectroscopy show that this infrared-bright source is a strongly star-forming pair of interacting galaxies at $z=0.35$. Adding existing Spitzer and SDSS photometry, we perform a spectral energy distribution analysis of the pair, and find that it has a combined infrared luminosity of $L_{\mathrm{IR}}=1.5 \times 10^{12} L_{\odot}$, comparable to other intermediate-redshift ultra-luminous infrared galaxies studied with Herschel. Isolating the emission from 3C 318's host, we robustly constrain the level of star formation to a value a factor of three lower than that published earlier, which is more in line with the star formation activity found in other Herschel-detected 3CR objects at similar redshift.
\end{abstract}

Key words. galaxies: active - galaxies: high-redshift - galaxies: star formation - infrared: galaxies - quasars: individual: 3C 318

\section{Introduction}

Powerful, high-redshift, radio-loud active galactic nuclei (AGN) provide a unique opportunity to study the interplay between the growth of the black hole and that of the host galaxy when both processes went through their peak activity.

The Revised Third Cambridge Catalogue of radio sources (hereafter 3CR; Bennett 1962; Spinrad et al. 1985) contains some of the most luminous radio galaxies (RGs) and quasars (QSRs) in the high- $z$ Universe, which have been studied with virtually all space telescopes (e.g. Best et al. 1998; Haas et al. 2008; Leipski et al. 2010; Wilkes et al. 2013; Chiaberge et al. 2015). Podigachoski et al. (2015, hereafter P15) recently performed a comprehensive study of this sample using far-infrared (FIR) data from the Herschel Space Observatory (Pilbratt et al. 2010), covering the last remaining spectral window in the study of these landmark objects. In that work, we found evidence for strong star formation (SF) activity in about $40 \%$ of $3 \mathrm{CR}$ hosts, at the level of several hundred solar masses per year, comparable to the SF activity in equally massive, non-AGN hosts at similar redshift.

3C 318 (Spinrad \& Smith 1976) is a QSR at a spectroscopically measured redshift of $z=1.574$ (Willott et al. 2000). It is a compact steep-spectrum (CSS), presumably young radio source, with a projected radio extent of $\sim 7 \mathrm{kpc}$ (Mantovani et al. 2010). Adopting a typical speed of radio-jet expansion of about $10 \%$ of the speed of light suggests that the jets were triggered $\sim 0.1 \mathrm{Myr}$ ago. 3C 318 is one of the highest redshift sources detected with the IRAS telescope (Hes et al. 1995), and is also detected with SCUBA at $850 \mu \mathrm{m}$ (Willott et al. 2002). These two strong detections suggested that 3 C 318 is unusually bright in the infrared. Given the steep-spectrum nature of the radio source, it is clear that the dominant emission in the infrared is thermal dust emission in its host galaxy. Taking into account the AGN contribution to the $850 \mu \mathrm{m}$ flux, estimated from the synchrotron spectral slope, Willott et al. (2007) reported a hyper-luminous star formation rate (SFR) of $1700 M_{\odot} \mathrm{yr}^{-1}$. Heywood et al. (2013) targeted the ground-state $\mathrm{CO}$ line in $3 \mathrm{C} 318$, and found that the molecular gas with an estimated mass of $M_{\mathrm{H}_{2}} \sim 3.7( \pm 0.4) \times$ $10^{10}\left(\alpha_{\mathrm{CO}} / 0.8\right) M_{\odot}$ is spatially offset from the position of the QSR. These authors argued that 3C 318 is either undergoing a major merger (whereby the starburst is taking place in a nearby merging galaxy), or is a highly-disturbed system. This amount of molecular gas, combined with the estimated SFR, results in a gas-depletion time scale of about $20 \mathrm{Myr}$ in this system.

As already noted by Spinrad \& Smith (1976), a faint pair of interacting galaxies is visible in their optical image ${ }^{1}$ about $20^{\prime \prime}$ west of 3C 318. The $r$-band SDSS-field of 3C 318 is shown in Fig. 1, with the position of 3C 318 and the galaxy pair (East and West) marked. The pair emits prominently in the Herschel maps published by P15. Given the poor angular resolution of IRAS, it is clear that the pair contributes significantly to the measured IRAS flux. Here, we assess the nature of the interacting galaxies by spectroscopically measuring their redshifts, and analysing their spectral energy distributions (SEDs). Using this newly acquired information, we update the SED of 3C 318's host, and modify several physical properties related to this peculiar object.

\section{Existing and new observations of $3 C 318$ and the nearby galaxy pair}

3C 318 is one of the strongest submillimetre (SCUBA) sources $\left(F_{850 \mu \mathrm{m}}=7.8 \pm 1.0 \mathrm{mJy}\right)$ detected by Willott et al. (2002). Given

\footnotetext{
1 Note that the scale provided with their optical image is incorrect, and that their redshift of 3C 318 was later updated to be $z=1.574$ (Willott et al. 2000).
} 


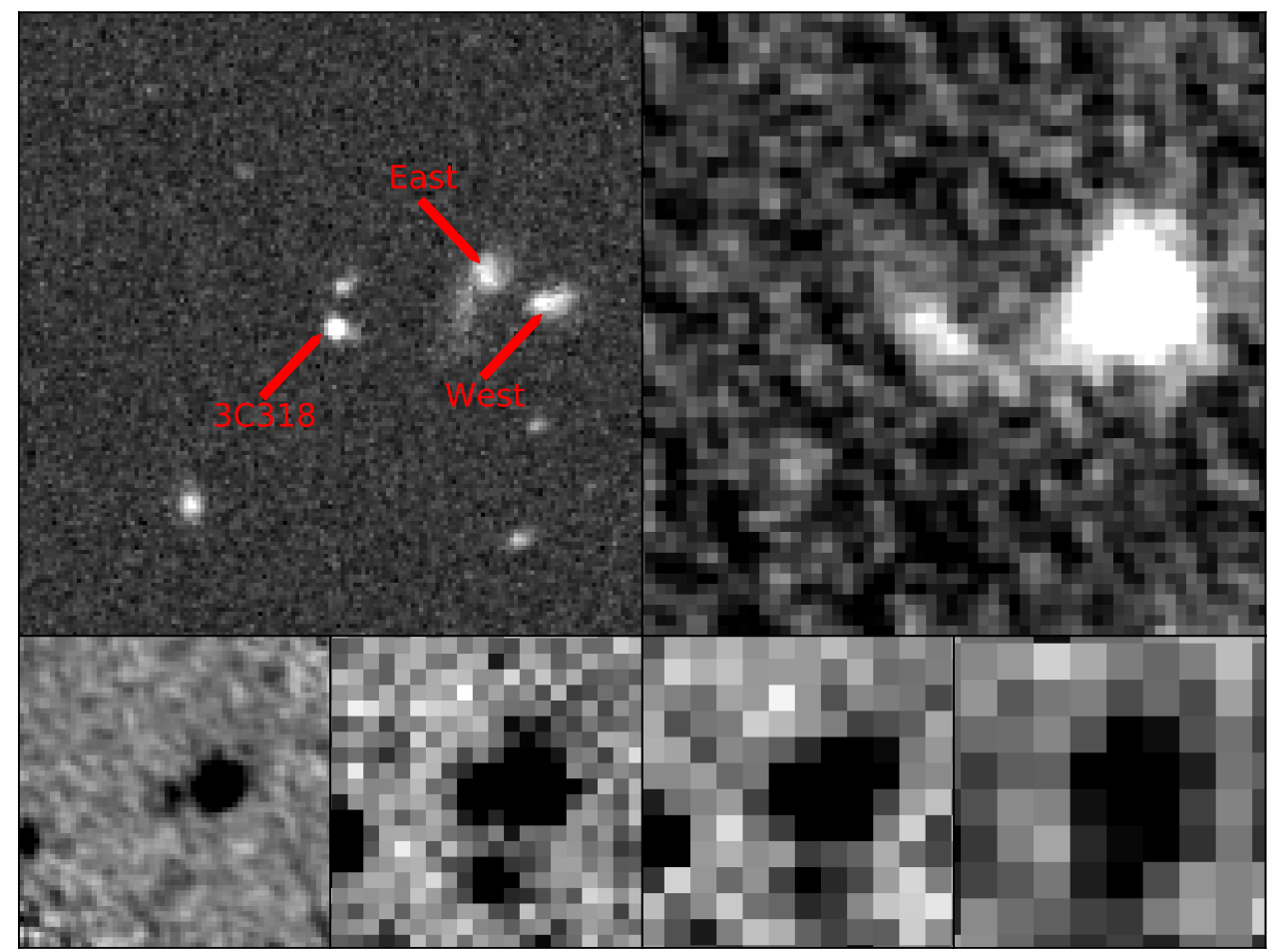

Fig. 1. 3C 318 and the nearby pair of interacting galaxies. Top left: SDSS $r$-band image of 3C 318's field, showing 3C 318 and the East and West galaxies of the nearby pair. Top right: PACS $70 \mu \mathrm{m}$ image showing 3C 318's host and the infrared-bright nearby pair. Each field is $1 \times 1$ arcmin in size and centered on 3C 318. Note that both the optical and the infrared images indicate the presence of additional objects close (in projection) to 3C 318. Lower panels: from left to right, Herschel maps in PACS $160 \mu \mathrm{m}$, SPIRE $250 \mu \mathrm{m}, 350 \mu \mathrm{m}$, and $500 \mu \mathrm{m}$, respectively. These maps are $2 \times 2$ arcmin in size and centered on 3C 318 .

that it is also detected with MAMBO $\left(F_{1.25} \mathrm{~mm}=5.6 \pm 1.4 \mathrm{mJy}\right.$; Haas et al. 2006), it is clear that part of the SCUBA flux is contributed by non-thermal (synchrotron) emission from 3C 318's radio core. Extrapolating from core radio data, Haas et al. (2006) estimated this contribution to be $\sim 3.5 \mathrm{mJy}$, which means that about $4.3 \mathrm{mJy}$ are due to thermal radiation from dust in 3C 318's host.

3C 318 and its surroundings were observed with the Spitzer Space Telescope (Werner et al. 2004) with all three imaging instruments: IRAC at 3.6, 4.5, 5.8, and $8.0 \mu \mathrm{m}$, IRS-16 peak-up array at $16 \mu \mathrm{m}$, and MIPS at $24 \mu \mathrm{m}$. Details of the data reduction and photometry were published by Haas et al. (2008). The photometry is presented in Table 1.

The Herschel observations of the field around 3C 318 were obtained as part of our guaranteed time project: the Herschel Legacy of distant radio-loud AGN (PI: Barthel). Five-band photometric measurements were carried out using both imaging instruments, PACS (Poglitsch et al. 2010) at 70 and $160 \mu \mathrm{m}$, and SPIRE (Griffin et al. 2010) at 250, 350, and $500 \mu \mathrm{m}$. Data reduction was performed as described by P15. In Fig. 1, 3C 318 can be clearly seen in the final PACS and SPIRE $250 \mu \mathrm{m}$ maps, but the interacting galaxies are unresolved, and deblending of the pair is not possible. The galaxy pair and 3C 318 fall within the same beam in the 350 and $500 \mu \mathrm{m}$ SPIRE bands. While 3C 318 can still have significant emission in the SPIRE 350 and $500 \mu \mathrm{m}$ bands, it is evident that most of the FIR emission comes from the galaxy pair. The 3C 318 flux densities in the PACS bands were obtained as outlined in P15, using apertures of $4^{\prime \prime}$ and $6^{\prime \prime}$ radius, for PACS $70 \mu \mathrm{m}$ and PACS $160 \mu \mathrm{m}$, respectively. Integrated PACS flux densities for the galaxy pair were measured using a $6^{\prime \prime}$ and $12^{\prime \prime}$ aperture in PACS $70 \mu \mathrm{m}$ and $160 \mu \mathrm{m}$, respectively. SPIRE photometry followed the procedure adopted by P15. Given the steep radio spectrum of 3C 318, no synchrotron contribution is expected in the SPIRE bands. All flux densities measured from the Herschel bands are listed in Table 1.

To determine the redshift of the pair of galaxies, and hence quantify the contribution of 3 C 318 to the integrated flux density in the 350 and $500 \mu \mathrm{m}$ SPIRE bands, we obtained optical spectra for each of the two galaxies with the ACAM instrument on the William Herschel Telescope (WHT) on February 7, 2014. The long-slit spectra were reduced using standard packages within IRAF. Identifying emission lines from [O II], $\mathrm{H} \beta$, [O III], and $\mathrm{H} \alpha$ in the noisy spectra, we measure the redshift for each galaxy to be $z=0.35 \pm 0.01$, with a relative velocity difference of $\sim 300 \mathrm{~km} \mathrm{~s}^{-1}$. Additional optical data (ugriz bands) for the pair of interacting galaxies were obtained from $\operatorname{SDSS}^{2}$. The flux densities in the optical bands are also listed in Table 1.

\section{Results and discussion}

The availability of photometric data over a large wavelength range allows us to obtain reasonable estimates of the galaxies' physical properties using an SED fitting approach. To this end, we use the code MAGPHYS ${ }^{3}$, which follows the approach outlined by da Cunha et al. (2008). This largely empirical (but physically motivated) code interprets the infrared SEDs of galaxies consistently with the emission at shorter wavelengths assuming (1) that only starlight heats the dust and; (2) that the luminosity absorbed by dust is re-emitted in the infrared domain. For details related to the adopted stellar populations and dust attenuation we refer the reader to da Cunha et al. (2008). Using $\chi^{2}$ minimization, the code constrains several physical properties related to the stars and the interstellar medium of the probed galaxies. Figure 2 shows the best-fit SEDs for the two interacting galaxies as determined by MAGPHYS using the SDSS and Spitzer photometry listed in Table 1, and the redshift of the interacting pair determined from the optical spectra. The fits are remarkably good despite the lack of constraints in the FIR domain, and both galaxies are best fitted with dusty starburst templates. Both galaxies are characterized by a considerable amount of dust attenuation and have their IRAC $8.0 \mu \mathrm{m}$ and IRS $16 \mu \mathrm{m}$ fluxes

\footnotetext{
2 http://www.sdss.org/dr12/

Publicly available at http://www.iap.fr/magphys/magphys/ MAGPHYS . html
} 
P. Podigachoski et al.: The far-infrared emission of the radio-loud quasar 3C $318(R N)$

Table 1. Photometry of 3C 318 and of the individual galaxies constituting the interacting pair close (in projection) to $3 \mathrm{C} 318$.

\begin{tabular}{cccccc}
\hline \hline Band & 3C 318 & East galaxy & West galaxy & Galaxy pair & 3C 318 and pair \\
\hline SDSS $u[\mu \mathrm{Jy}]$ & $\ldots$ & $3 \pm 2$ & $6 \pm 2$ & $\ldots$ & $\ldots$ \\
SDSS $g[\mu \mathrm{Jy}]$ & $\ldots$ & $26 \pm 1$ & $15 \pm 7$ & $\ldots$ & $\ldots$ \\
SDSS $r[\mu \mathrm{Jy}]$ & $\ldots$ & $72 \pm 2$ & $48 \pm 1$ & $\ldots$ & $\ldots$ \\
SDSS $i[\mu \mathrm{Jy}]$ & $\ldots$ & $108 \pm 2$ & $77 \pm 1$ & $\ldots$ & $\ldots$ \\
SDSS $z[\mu \mathrm{Jy}]$ & $\ldots$ & $141 \pm 10$ & $112 \pm 7$ & $\ldots$ & $\ldots$ \\
IRAC $3.6[\mu \mathrm{Jy}]$ & $343 \pm 51$ & $247 \pm 24$ & $289 \pm 28$ & $\ldots$ & $\ldots$ \\
IRAC $4.5[\mu \mathrm{Jy}]$ & $427 \pm 64$ & $238 \pm 23$ & $293 \pm 29$ & $\ldots$ & $\ldots$ \\
IRAC $5.8[\mu \mathrm{Jy}]$ & $571 \pm 86$ & $212 \pm 21$ & $315 \pm 31$ & $\ldots$ & $\ldots$ \\
IRAC $8.0[\mu \mathrm{Jy}]$ & $806 \pm 121$ & $540 \pm 54$ & $933 \pm 93$ & $\ldots$ & $\ldots$ \\
IRS $16[\mu \mathrm{Jy}]$ & $1960 \pm 294$ & $540 \pm 54$ & $1500 \pm 150$ & $\ldots$ & $\ldots$ \\
MIPS $24[\mu \mathrm{Jy}]$ & $3400 \pm 510$ & $1140 \pm 114$ & $3000 \pm 300$ & $\ldots$ & $\ldots$ \\
PACS $70[\mathrm{mJy}]$ & $17.2 \pm 1.8$ & 58 & 163 & $221.2 \pm 3.8$ & $\ldots$ \\
PACS $160[\mathrm{mJy}]$ & $38.3 \pm 4.2$ & 73 & 205 & $277.5 \pm 6.1$ & $\ldots$ \\
SPIRE $250[\mathrm{mJy}]$ & $35.9 \pm 6.1$ & 43 & 120 & $163.4 \pm 6.1$ & $\ldots$ \\
SPIRE $350[\mathrm{mJy}]$ & 11 & $\ldots$ & $\ldots$ & $\ldots$ & $91.3 \pm 6.2$ \\
SPIRE $500[\mathrm{mJy}]$ & 16 & $\ldots$ & $\ldots$ & $\ldots$ & $46.6 \pm 6.3$ \\
SCUBA $850[\mathrm{mJy}]$ & 4.3 & $\ldots$ & $\ldots$ & $\ldots$ & $\ldots$ \\
\hline
\end{tabular}

Notes. The East (15:20:04.45, +20:16:10.55) and West (15:20:04.05, +20:16:07.91) galaxies are unresolved in the PACS $70 \mu \mathrm{m}$, PACS 160 $\mu \mathrm{m}$, and SPIRE $250 \mu \mathrm{m}$ bands. The Herschel PACS and SPIRE OBSIDs are 1342223844/1342223845 and 1342204107, respectively. Both galaxies and 3C 318 are within the same beam in the SPIRE $350 \mu \mathrm{m}$ and SPIRE $500 \mu \mathrm{m}$ bands. Estimated photometry (as explained in the text) is given in italics.

Table 2. MAGPHYS best-fit physical properties.

\begin{tabular}{ccccc}
\hline \hline & $\begin{array}{c}M_{\text {stellar }} \\
{\left[M_{\odot}\right]}\end{array}$ & $\begin{array}{c}L_{\mathrm{IR}} \\
{\left[L_{\odot}\right]}\end{array}$ & $\begin{array}{c}M_{\text {dust }} \\
{\left[M_{\odot}\right]}\end{array}$ & $\chi^{2}$ \\
\hline East galaxy & $5.0 \times 10^{10}$ & $3.9 \times 10^{11}$ & $1.6 \times 10^{8}$ & 2.7 \\
West galaxy & $8.0 \times 10^{10}$ & $1.1 \times 10^{12}$ & $4.6 \times 10^{8}$ & 1.3 \\
\hline
\end{tabular}

dominated by emission from polycyclic aromatic hydrocarbon features. As a result from the low signal-to-noise $u$-band photometry for the East galaxy, its best-fit is slightly worse than that of the West galaxy. No differences in the derived paramaters were found when performing the fit without taking this data point into account. The results, listed in Table 2, suggest that relative to the East galaxy, the West galaxy has a factor of 1.6 higher stellar mass $\left(M_{\text {stellar }}\right)$, and a factor of 2.8 higher infrared luminosity $\left(L_{\mathrm{IR}}\right)$. We adopt the $L_{\mathrm{IR}}$ ratio between the two galaxies, and use it to estimate the contribution of each individual galaxy to the integrated flux density measured in the three shortest Herschel bands (see Table 1). We perform additional fits using these estimated flux densities in addition to the SDSS and Spitzer flux densities, and find similar physical properties (within 10\%) confirming that the fits obtained without the Herschel constraints are remarkably robust. For completeness, we overplot the estimated Herschel photometry in Fig. 2 (green points). The morphology and size of the interacting pair of galaxies are comparable to those of the well-known Antennae (NGC 4038/NGC 4039, e.g. Whitmore \& Schweizer 1995), which have a total FIR luminosity of $L_{\mathrm{FIR}} \sim 1.3 \times 10^{11} L_{\odot}$ (Klaas et al. 2010). The total infrared luminosity of the pair is in line with Herschel-studied ULIRGs at $0.2<z<0.9$ (Magdis et al. 2014).

Willott et al. (2000) presented the hitherto obtained radio to X-ray SED of 3C 318 and determined that its infrared
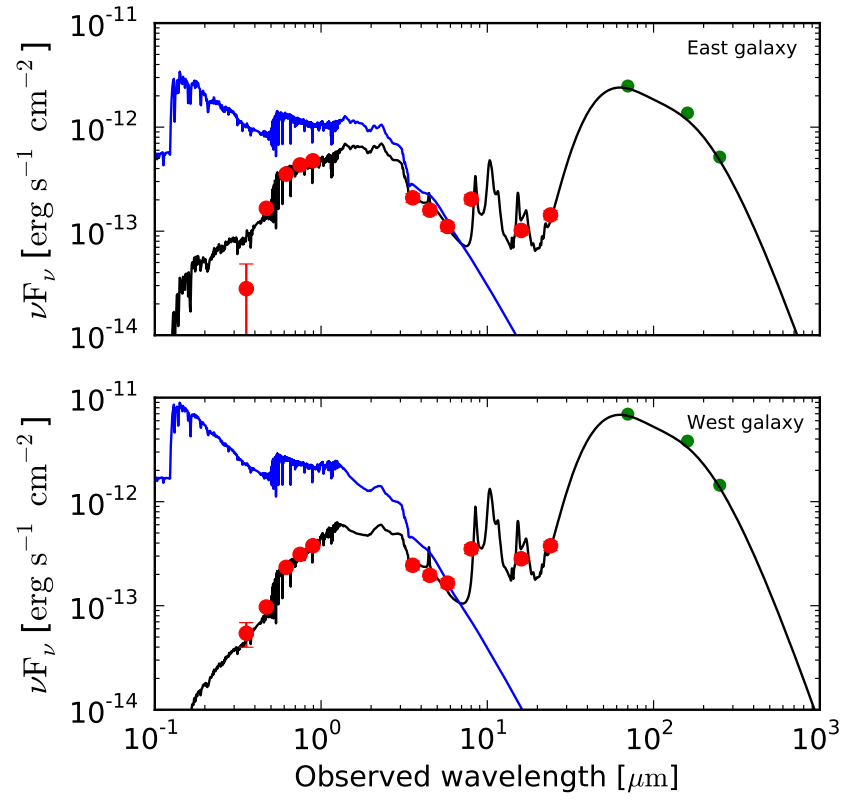

Fig. 2. MAGPHYS best-fit spectral energy distributions (SEDs) of the interacting pair close (in projection) to 3C 318. Top: the East galaxy. Bottom: the West galaxy. The observed (Spitzer and SDSS) photometry is listed in Table 1, and plotted with red circles. The blue and black solid lines, respectively, correspond to the unattenuated and attenuated bestfit SEDs. Green circles represent the photometry at the three shortest Herschel bands, estimated using the computed infrared luminosity ratio and the measured integrated Herschel flux density (see text).

luminosity, $L_{\mathrm{FIR}}$, greatly exceeds $10^{13} L_{\odot}$. Correcting for the non-thermal core emission, Willott et al. (2007) modified it to a value of $L_{\mathrm{FIR}} \sim 10^{13} L_{\odot}$, placing $3 \mathrm{C} 318$ at the border between what is known in the literature as ultra-luminous (ULIRG) 


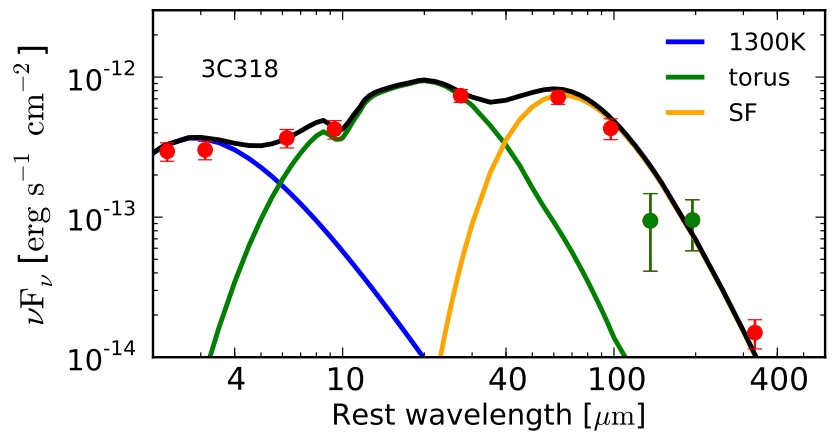

Fig. 3. Best-fit infrared spectral energy distribution (SED) of the quasar 3C 318. Red circles represent the observed photometry, and green circles the estimated photometry (see text). The total SED (black) is given as the sum of AGN-heated hot dust close to sublimation temperature (blue), of torus emission (green, from the library of Hönig \& Kishimoto 2010), and of star-formation-heated dust (yellow).

and hyper-luminous (HyLIRG) infrared galaxies. Using better quality Spitzer and Herschel data, P15 fitted the infrared SED of 3C 318 obtaining $L_{\mathrm{SF}}=3.4_{-0.3}^{+0.4} \times 10^{12} L_{\odot}$, firmly establishing the ULIRG nature of $3 \mathrm{C} 318$.

Here we revisit the infrared SED of 3C 318 using the just obtained SPIRE 350 and $500 \mu \mathrm{m}$ photometry. To isolate the signal from 3C 318, we use the best-fits for the pair galaxies determined by MAGPHYS, extrapolate the flux densities at these two bands, and finally subtract them from the integrated values given in Table 1. Following P15, we fit 3C 318's infrared SED using the sum of three components accounting for the emission from AGN-heated hot dust close to sublimation temperature (represented by a blackbody with a fixed temperature of $1300 \mathrm{~K}$ ), the AGN-heated warm dust (torus) emission (represented by a model from the library of Hönig \& Kishimoto 2010), and the SF-heated dust on the scale of the host galaxy (represented by a modified blackbody with a fixed emissivity index).

The best-fit SED, presented in Fig. 3, represents the photometric data points well, except for the SPIRE $350 \mu \mathrm{m}$ point. Integrating under the $\mathrm{AGN}$ and $\mathrm{SF}$ related components gives $L_{\mathrm{AGN}}=7.6 \times 10^{12} L_{\odot}$ and $L_{\mathrm{SF}}=3.4 \times 10^{12} L_{\odot}$, comparable to what was found for other SPIRE detected sources from the same $3 \mathrm{CR}$ catalogue (P15). The $L_{\mathrm{SF}}$ value obtained here is a factor of three lower than that determined by Willott et al. (2007). Consequently, the gas-depletion time scale (or SF efficiency) of 3C 318 is a factor of three longer than that computed by Heywood et al. (2013) based on a molecular gas mass of $M_{\mathrm{H}_{2}} \sim 3.7( \pm 0.4) \times 10^{10}\left(\alpha_{\mathrm{CO}} / 0.8\right) M_{\odot}$, and is about $60 \mathrm{Myr}$. These numbers place $3 \mathrm{C} 318$ well below the $L_{\mathrm{FIR}}-L_{\mathrm{CO}}^{\prime}$ relation for high- $z$ submillimetre galaxies reported by Bothwell et al. (2013), but still imply strong SF activity in its host galaxy. Note that part of the measured infrared flux density in the Herschel bands could also be attributed to the close companion southwest of 3C 318, noted by Willott et al. (2000) and seen in Fig. 1. Whether this companion is at the same redshift as $3 \mathrm{C} 318$, and possibly interacting with it, remains to be seen. Future ALMA studies will likely provide an improved view of the dust and molecular gas emission surrounding 3C 318.

\section{Conclusion}

Herschel imaging of the field centered on the radio-loud quasar 3C 318 reveals that most of the infrared emission detected with earlier instruments, and associated with 3C 318, comes from a pair of interacting galaxies close (in projection) to $3 \mathrm{C} 318$. Using optical spectroscopy, we determine the redshift of the pair to be $z=0.35$. We perform a spectral energy distribution analysis of the pair, and constrain its infrared luminosity to $L_{\mathrm{IR}}=1.5 \times$ $10^{12} L_{\odot}$, comparable to the luminosity of other ultra-luminous infrared galaxies at that redshift. We quantify the emission of 3C 318's host galaxy in Herschel bands where it is blended with the galaxy pair, and find that its luminosity due to SF activity is $L_{\mathrm{SF}}=3.4 \times 10^{12} L_{\odot}$, a factor of three lower than found in previous studies. Further ALMA studies are needed to get an improved understanding of the dust emission in 3C 318's host.

Acknowledgements. P.P. acknowledges the Nederlandse Organisatie voor Wetenschappelijk Onderzoek (NWO) for a Ph.D. fellowship. The authors acknowledge Javier Mendez for obtaining the optical spectroscopy, Steve Willner for providing the Spitzer photometry, Scott Trager for helping with the redshift determination, and Wouter Karman for useful comments. Herschel is an ESA space observatory with science instruments provided by European-led Principal Investigator consortia and with important participation from NASA. This work is partly based on observations made with the Spitzer Space Telescope, which is operated by the Jet Propulsion Laboratory, California Institute of Technology under a contract with NASA. Funding for the Sloan Digital Sky Survey IV has been provided by the Alfred P. Sloan Foundation and the Participating Institutions. SDSS-IV acknowledges support and resources from the Center for High-Performance Computing at the University of Utah. The SDSS web site is http://www.sdss.org. Based on observations made with the William Herschel Telescope (WHT) operated on the island of La Palma by the Isaac Newton Group in the Spanish Observatorio del Roque de los Muchachos of the Instituto de Astrofísica de Canarias. This research made use of APLpy, an opensource plotting package for Python hosted at http://aplpy.github.com.

\section{References}

Bennett, A. S. 1962, MmRAS, 68, 163

Best, P. N., Longair, M. S., \& Roettgering, H. J. A. 1998, MNRAS, 295, 549 Bothwell, M. S., Smail, I., Chapman, S. C., et al. 2013, MNRAS, 429, 3047

Chiaberge, M., Gilli, R., Lotz, J. M., \& Norman, C. 2015, ApJ, 806, 147

da Cunha, E., Charlot, S., \& Elbaz, D. 2008, MNRAS, 388, 1595

Griffin, M. J., Abergel, A., Abreu, A., et al. 2010, A\&A, 518, L3

Haas, M., Chini, R., Müller, S. A. H., Bertoldi, F., \& Albrecht, M. 2006, A\&A, 445,115

Haas, M., Willner, S. P., Heymann, F., et al. 2008, ApJ, 688, 122

Hes, R., Barthel, P. D., \& Hoekstra, H. 1995, A\&A, 303, 8

Heywood, I., Martínez-Sansigre, A., Willott, C. J., \& Rawlings, S. 2013, MNRAS, 435, 3376

Hönig, S. F., \& Kishimoto, M. 2010, A\&A, 523, A27

Klaas, U., Nielbock, M., Haas, M., Krause, O., \& Schreiber, J. 2010, A\&A, 518, L44

Leipski, C., Haas, M., Willner, S. P., et al. 2010, ApJ, 717, 766

Magdis, G. E., Rigopoulou, D., Hopwood, R., et al. 2014, ApJ, 796, 63

Mantovani, F., Rossetti, A., Junor, W., Saikia, D. J., \& Salter, C. J. 2010, A\&A, 518, A33

Pilbratt, G. L., Riedinger, J. R., Passvogel, T., et al. 2010, A\&A, 518, L1

Podigachoski, P., Barthel, P. D., Haas, M., et al. 2015, A\&A, 575, A80 (P15)

Poglitsch, A., Waelkens, C., Geis, N., et al. 2010, A\&A, 518, L2

Spinrad, H., \& Smith, H. E. 1976, ApJ, 206, 355

Spinrad, H., Marr, J., Aguilar, L., \& Djorgovski, S. 1985, PASP, 97, 932

Werner, M. W., Roellig, T. L., Low, F. J., et al. 2004, ApJS, 154, 1

Whitmore, B. C., \& Schweizer, F. 1995, AJ, 109, 960

Wilkes, B. J., Kuraszkiewicz, J., Haas, M., et al. 2013, ApJ, 773, 15

Willott, C. J., Rawlings, S., \& Jarvis, M. J. 2000, MNRAS, 313, 237

Willott, C. J., Rawlings, S., Archibald, E. N., \& Dunlop, J. S. 2002, MNRAS, 331,435

Willott, C. J., Martínez-Sansigre, A., \& Rawlings, S. 2007, AJ, 133, 564 